\title{
Serum Branched Chain Amino Acids Are Associated with Type 2 Diabetes Mellitus in Jordan
}

\author{
Mahmoud A. Alfaqih*, Zaina Abu-Khdair, Rami Saadeh, Nesreen Saadeh, Ahmed Al-Dwairi, Othman Al-Shboul \\ Faculty of Medicine, Jordan University of Science and Technology, Irbid, Jordan
}

Background: Diabetes mellitus is a global public health problem that is caused by the lack of insulin secretion (type 1) or resistance to its action (type 2). A low insulin-to-glucagon ratio predicts an increase in the serum levels of branched chain amino acids, a feature confirmed in several populations. This relationship has not been assessed in Jordan. The objective of this study was to investigate the association between serum branched chain amino acids and type 2 diabetes mellitus in patients in Jordan.

Methods: Two hundred type 2 diabetes mellitus patients and an additional 200 non-diabetic controls were recruited. Age, body mass index, and waist circumference of the subjects were recorded. Branched chain amino acid, total cholesterol, and triglyceride levels were measured from the collected serum samples.

Results: Serum branched chain amino acid levels were significantly higher in type 2 diabetes mellitus patients than in non-diabetes individuals $(\mathrm{P}<0.0001)$. In binomial regression analysis, serum branched chain amino acid levels remained significantly associated with diabetes mellitus and increased its risk (odds ratio, 1.004; 95\% confidence interval, 1.001-1.006; $\mathrm{P}=0.003$ ).

Conclusion: Type 2 diabetes mellitus is associated with higher branched chain amino acid levels in Jordan independent of age, sex, body mass index, waist circumference, and total serum cholesterol and serum triglyceride levels.

Keywords: Diabetes Mellitus; Branched Chain Amino Acids; Body Mass Index

Received: October 30, 2017, Revised: January 4, 2018, Accepted: January 11, 2018

${ }^{*}$ Corresponding Author: Mahmoud A. Alfaqih https://orcid.org/0000-0002-6383-7014

Tel: +962-2-7201000, Fax: +962-2-7095123, E-mail: maalfaqih@just.edu.jo 


\section{INTRODUCTION}

Diabetes mellitus (DM) is a worldwide health problem of epidemic dimensions. In 2015, 415 million adults were diagnosed with DM worldwide, with a prevalence of $8.8 \% .{ }^{1)}$ Noteworthy, there is a clear geographic disparity in the prevalence of DM, with about $75 \%$ of patients living in low- and middle-income countries. ${ }^{1)}$ The global prevalence of DM has increased from $4.7 \%$ in 1980 to $8.5 \%$ in $2014 .^{2)}$ This increase was most rapid in low- and middle-income countries, with the highest national prevalence rates registered in Oceania and the Middle East and North Africa (MENA) regions. ${ }^{2)}$ Most of the diabetes patients fall into either of two broad categories, namely type $1 \mathrm{DM}$ (T1DM) or type $2 \mathrm{DM}$ (T2DM), with T2DM being more prevalent. ${ }^{3)} \mathrm{T} 1 \mathrm{DM}$ is characterized by the complete absence of insulin secretion, while T2DM is caused by resistance to insulin action on target tissues with inadequate blood insulin levels. ${ }^{3,4)} \mathrm{DM}$ places a large financial burden on individuals and healthcare systems; global estimates for health expenditure due to DM was approximately 673 billion US dollars (USD) in 2015, and this figure is expected to reach 802 billion USD by $2040{ }^{5)}$ In the MENA region alone, the health expenditure was approximately 17.1 billion USD in 2015 and is expected to double by $2040 .^{5)}$

Jordan is a developing country in the MENA region, and the most recent statistics indicated that over a period of 10 years (1994-2004), the prevalence of DM in Jordan has increased from $13 \%$ to $17 \%{ }^{6}{ }^{6}$ The exact cause of the T2DM epidemic in Jordan is not understood; however, this could be related to a changing lifestyle that is characterized by the lack of exercise and the adoption of a western diet. ${ }^{7,8}$ Nonetheless, the high number of T2DM cases in Jordan highlights the need for proper preventative and interventional measures to reduce the economic and health burden of this epidemic.

Branched chain amino acids (BCAAs) are a group of essential amino acids, which include leucine, isoleucine, and valine. ${ }^{9)}$ An elevation in serum BCAA levels in diabetes patients was reported as early as 1970 when Felig et al. ${ }^{10)}$ observed an increase in serum BCAA levels in diabetes patients with ketoacidosis. This observation, however, did not receive attention until recently when several studies showed an increase in serum BCAA levels in T1DM and T2DM patients in addition to other conditions linked to insulin resistance. ${ }^{11,12)}$ Notably, despite the economic and health burden of T2DM in the MENA region (including Jordan), there are no studies that investigated serum BCAA levels in diabetes patients, or their relationship to co-morbidities or other markers of insulin resistance. Herein, using a case-control study design, we investigated the relationship between serum BCAA levels and T2DM in patients at a tertiary institution of Northern Jordan.

\section{METHODS}

\section{Study Design}

A case-control design was used in this investigation. Approval to recruit subjects to participate was granted by the Institutional Review Boards of the Jordan University of Science and Technology located in
Irbid in the northern part of Jordan (IRB approval no., 249/2016). Signature on a consent form was a prerequisite for participation. Recruitment of study subjects took place between August 2016 and April 2017 at the clinics of King Abdullah University Hospital, a tertiary hospital affiliated with Jordan University of Science and Technology. This hospital is currently the largest hospital in the northern part of Jordan in terms of its capacity, number of staff members, and number of patients that visit the hospital on a daily basis. This hospital serves residents of the following governorates: Irbid, Ajloun, Jerash, and Mafraq, which together totaled one million inhabitants. The hospital has a bed capacity of 683 that can be increased to 800 beds in emergency cases.

\section{Subject Description}

Four hundred subjects with an age range of 35-70 years were invited to participate in this investigation. Two hundred subjects were previously diagnosed with T2DM by two endocrinologists according to the American Diabetes Association guidelines. ${ }^{3)}$ All the diabetes cases were recruited from the endocrinology clinics at King Abdullah University Hospital. The remaining 200 subjects did not have any symptoms of T2DM at the time of the investigation and were recruited from the same tertiary hospital though not necessarily from the endocrinology clinics. Absence of diabetes in the control arm of the study was assessed through the withdrawal of blood following an overnight fast and the measurement of fasting blood glucose (FBG) on two separate occasions. Pre-diabetes subjects with a repeated FBG of 100-125 mg/dL were excluded from participating in this study. Subjects with Cushing's syndrome, polycystic ovarian syndrome, thyroid dysfunction, or hyperprolactinemia were also excluded from both arms of the study. Subjects who were taking BCAA supplements were also excluded from both arms of the study. Non-diabetes subjects were selected by matching body mass index (BMI) and age to diabetes patients. Diabetes patients were grouped into the following BMI categories: $<25.0 \mathrm{~kg} / \mathrm{m}^{2}$, $25.0-29.9 \mathrm{~kg} / \mathrm{m}^{2}$, or $\geq 30.0 \mathrm{~kg} / \mathrm{m}^{2}$, and the following age categories: $\leq 40$ years, $41-50$ years, $51-60$ years, and $61-70$ years. Frequency matching was then performed during the recruitment of non-diabetes subjects.

\section{Anthropometric Measurements}

The participants' height $(\mathrm{cm})$ and weight $(\mathrm{kg})$ were recorded at the time of their visit to King Abdullah University Hospital. BMI was calculated based on the above measurements using the following equation: BMI=weight $(\mathrm{kg}) /$ height $^{2}\left(\mathrm{~m}^{2}\right)$. The waist circumference (WC) was measured in centimeters $(\mathrm{cm})$ using a tape measure positioned at the midpoint between the inferior costal margin and the superior border of the iliac crest with the measurement site being at the level of the umbilicus.

\section{Blood Sampling and Handling}

After a 12-hour fast, $10 \mathrm{~mL}$ of blood was withdrawn from the study subjects in a plain tube containing a clot activator (AFCO, Amman, Jordan). Serum was obtained from the samples by centrifuging at 3,000 $\times \mathrm{g}$ for 5 minutes. The serum samples were later used to measure glu- 
cose, total cholesterol, triglyceride, and BCAA levels.

\section{Biochemical Measurements}

Serum BCAA levels were measured using a commercially available kit purchased from Sigma Aldrich (St. Louis, MO, USA) with leucine as the standard. The assay involves a coupled enzymatic reaction to determine the levels of all three BCAAs, resulting in a colorimetric product. The absorbance was measured using the ELx800 Microplate Reader (BioTek Instruments, Winooski, VT, USA). A wavelength of 450 $\mathrm{nm}$ was used for the measurements. Additionally, the serum samples were submitted to the laboratories of King Abdullah University Hospital to measure glucose, total cholesterol, and triglyceride levels using an automated analyzer system (Roche Diagnostics, Mannheim, Germany).

\section{Statistical Analysis}

Statistical analyses were performed using the IBM SPSS software ver. 22.0 (IBM Corp., Armonk, NY, USA). The Student t-test was used to test for significant differences in BCAA levels, age, sex (male or female), BMI, WC, and total cholesterol, triglyceride, or glucose levels between diabetes and non-diabetes subjects. Binomial logistic regression was used to test the association between BCAA levels and diabetes after including the following variables: age, sex, BMI, WC, and total cholester$\mathrm{ol}$ and triglyceride levels. A P-value of $<0.05$ was used as a cut-off for significance.

\section{RESULTS}

\section{Subject Characteristics and Biochemical Profile}

During the course of this study, 200 diabetes and 200 non-diabetes subjects were eligible. The percentage of females was higher than males among non-diabetes (58\%) and diabetes (51.5\%) subjects (Table 1); however, no significant differences existed in sex distribution between non-diabetes and diabetes subjects $(\mathrm{P}=0.218)$. Diabetes subjects were significantly older than non-diabetes $(57.81 \pm 10.18$ versus $52.24 \pm 10.28$ years, $\mathrm{P}<0.0001$ ) (Table 1 ). Furthermore, diabetes subjects had a higher $\mathrm{BMI}\left(30.82 \pm 5.35\right.$ versus $\left.29.78 \pm 5.24 \mathrm{~kg} / \mathrm{m}^{2}, \mathrm{P}=0.067\right)$ and a significantly larger WC $(109.59 \pm 12.66$ versus $102.27 \pm 12.25 \mathrm{~cm}$, $\mathrm{P}<0.0001$ ) (Table 1).

The biochemical profile of the subjects showed that diabetes subjects had significantly higher levels of fasting serum BCAAs (469.00 \pm 117.22 versus $418.71 \pm 113.72 \mu \mathrm{mol} / \mathrm{L}, \mathrm{P}<0.0001)$, glucose (167.67 \pm 82.59 versus $86.65 \pm 10.84 \mathrm{mg} / \mathrm{dL}, \mathrm{P}<0.0001)$, cholesterol $(208.93 \pm 69.78$ versus $185.92 \pm 40.06 \mathrm{mg} / \mathrm{dL}, \mathrm{P}<0.0001)$ and triglycerides (163.5 \pm 99.36 versus $140.82 \pm 105.24 \mathrm{mg} / \mathrm{dL}, \mathrm{P}<0.0001$ ) (Table 1).

\section{Association between Branched Chain Amino Acids and Diabetes}

Given that previous reports found that serum BCAA levels could be modified by BMI, ${ }^{13)}$ sex, ${ }^{14)}$ and $\mathrm{WC},{ }^{15)}$ we wanted to test whether serum BCAA levels remained associated with diabetes in our study popula-

Table 1. Baseline characteristics of diabetes patients and non-diabetes subjects

\begin{tabular}{lcc}
\multicolumn{1}{c}{ Characteristic } & Non-diabetes subjects $(\mathrm{N}=200)$ & Diabetes patients $(\mathrm{N}=200)$ \\
\hline Sex & & \\
Males & $81(41)$ & $96(48)$ \\
Females & $116(59)$ & $103(42)$ \\
Missing & 3 & 1 \\
Total & 200 & 200 \\
Age $(\mathrm{y})$ & $52.24 \pm 10.28$ & $57.81 \pm 10.18$ \\
Body mass index $\left(\mathrm{kg} / \mathrm{m}^{2}\right)$ & $29.78 \pm 5.24$ & $30.82 \pm 5.35$ \\
Waist circumference $(\mathrm{cm})$ & $102.27 \pm 12.25$ & $109.59 \pm 12.66$ \\
Branched chain amino acid $(\mu \mathrm{mol} / \mathrm{L})$ & $418.71 \pm 113.72$ & $469.00 \pm 117.22$ \\
Glucose $(\mathrm{mg} / \mathrm{dL})$ & $86.65 \pm 10.84$ & $167.67 \pm 82.59$ \\
Cholesterol $(\mathrm{mg} / \mathrm{dL})$ & $185.92 \pm 40.06$ & $<.0001$ \\
Triglyceride $(\mathrm{mg} / \mathrm{dL})$ & $140.82 \pm 105.24$ & $<0.0001$ \\
\hline
\end{tabular}

Values are presented as number (\%) or mean \pm standard deviation. P-values were calculated by the Student t-test.

Table 2. Binomial regression analysis of study participants with sex, age, BMI, waist circumference, BCAA, cholesterol level, and triglyceride level as variables

\begin{tabular}{lcc}
\hline \multicolumn{1}{c}{ Variable } & Estimate \pm standard error & Odds ratio (Wald 95\% confidence interval) \\
\hline Sex (female) & $0.100 \pm 0.284$ & $1.105(0.633-1.929)$ \\
Age $(\mathrm{y})$ & $0.028 \pm 0.013$ & $1.028(1.002-1.055)$ \\
BMl $\left(\mathrm{kg} / \mathrm{m}^{2}\right)$ & $-0.021 \pm 0.037$ & $0.979(0.911-1.053)$ \\
Waist circumference $(\mathrm{cm})$ & $0.038 \pm 0.015$ & 0.034 \\
BCAA $(\mu \mathrm{mol} / \mathrm{L})$ & $0.004 \pm 0.001$ & 0.572 \\
Cholesterol $(\mathrm{mg} / \mathrm{dL})$ & $0.008 \pm 0.003$ & $1.004(1.009-1.070)$ \\
Triglyceride $(\mathrm{mg} / \mathrm{dL})$ & $-0.001 \pm 0.001-1.006)$ & $1.008(1.003-1.013)$ \\
\hline
\end{tabular}

P-values were calculated by binomial logistic regression analysis.

BMl, body mass index; BCAA, branched chain amino acids. 
tion following adjustment with the above variables. Therefore, we used binomial logistic regression and found that serum BCAA levels significantly increased diabetes risk (odds ratio [OR], 1.004; 95\% confidence interval [CI], 1.001-1.006; $\mathrm{P}=0.003$ ) (Table 2) even in the presence of the above variables in the model. Noteworthy, total serum cholesterol showed the strongest association with diabetes in our model (OR, 1.008; 95\% CI, 1.003-1.013; P=0.002) (Table 2).

\section{DISCUSSION}

T2DM is a threat to public health worldwide in view of its epidemic nature and effect on a number of health outcomes. ${ }^{16)}$ T2DM increases the risk of developing atherosclerosis, ${ }^{17)}$ dyslipidemia, ${ }^{18)}$ and hypertension, ${ }^{19)}$ all of which are leading causes of death in both developed and developing countries. Owing to a rapidly changing lifestyle, the scale of this epidemic is of similar proportions in Jordan. ${ }^{6}$ Serum BCAA levels were shown to be elevated in a number of populations of various ethnic backgrounds. However, the association between serum BCAA levels and T2DM was not tested before in Jordan or any other country in the MENA region. Here, using a case-control study design, we showed that analogous to other populations, serum BCAA levels are increased in diabetes patients relative to non-diabetes control subjects. Control subjects in our study were selected by matching age and BMI to diabetes subjects. However, there was significant discrepancy in age between non-diabetes and diabetes subjects. This discrepancy was caused by mismatching between diabetes and non-diabetes in the 6170-year age category. Please look at the methods section for a description of the age categories used in the matching process. Regardless of the discrepancy, serum BCAA levels remained significantly associated with diabetes after adjusting for age, sex, BMI, WC, and total cholesterol and triglyceride levels in our logistic regression model.

Despite the fact that elevated serum BCAA levels are a consistent feature of T2DM in several populations, it remains to be determined whether the increase in serum BCAA levels is a cause of insulin resistance and/or T2DM or is a mere outcome of the disease. This question may be better answered using well-controlled experiments in cell and animal models. A review of the literature shows that there is evidence that supports both scenarios; for example, Newgard et al. ${ }^{20)}$ showed that feeding rats a high fat diet combined with BCAAs induced insulin resistance relative to mice fed normal chow. On the other hand, there was an increased breakdown of muscle protein and a consequent increase in serum BCAA levels in starved rats; ${ }^{21)}$ a metabolic state of low insulin-to-glucagon ratio analogous to insulin resistance. This indicates that elevation of serum BCAA levels could be an outcome of T2DM. Alternatively, given the above observations, an elevation in serum BCAA levels could be part of a vicious cycle where an initial increase in serum BCAA levels predisposes individuals to T2DM, which further increases BCAA levels as the disease progresses into later stages.

Noteworthy, the development of T2DM is preceded by a stage commonly referred to as "pre-diabetes" or "impaired glucose tolerance."22)
The importance of recognizing pre-diabetes individuals lies in the fact that proper intervention and/or lifestyle changes in pre-diabetes individuals may delay and/or prevent the progression of the disease. ${ }^{22)}$ Pre-diabetes individuals were excluded from participating in this study, a limitation of this investigation in view of the significance of finding biomarkers that help identify pre-diabetes individuals. BCAAs are the most abundant amino acids in the $\operatorname{diet}^{23)}$ and could be increased in individuals that consume high amounts of protein, ${ }^{23)}$ a potential confounding variable which we did not include in our study design and is yet another limitation of this investigation.

In conclusion, this study is the first to indicate an increase in serum BCAA levels in the MENA region. Future studies should attempt to correlate BCAA levels with the risk of developing "overt" diabetes in pre-diabetes individuals.

\section{CONFLICT OF INTEREST}

No potential conflict of interest relevant to this article was reported.

\section{ACKNOWLEDGMENTS}

Funding for this grant was provided through grant number (233/2016) awarded from the Deanship of Research at Jordan University of Science and Technology.

\section{REFERENCES}

1. Ogurtsova K, da Rocha Fernandes JD, Huang Y, Linnenkamp U, Guariguata L, Cho NH, et al. IDF diabetes atlas: global estimates for the prevalence of diabetes for 2015 and 2040. Diabetes Res Clin Pract 2017;128:40-50.

2. NCD Risk Factor Collaboration (NCD-RisC). Worldwide trends in diabetes since 1980: a pooled analysis of 751 population-based studies with 4.4 million participants. Lancet 2016;387:1513-30.

3. American Diabetes Association. 2. Classification and diagnosis of diabetes. Diabetes Care 2016;39(Suppl 1):S13-S22.

4. Ahima RS. Editorial: rethinking the definition of diabetes for precision medicine. Mol Endocrinol 2015;29:335-7.

5. International Diabetes Federation. IDF diabetes atlas. Brussels: International Diabetes Federation; 2015.

6. Ajlouni K, Khader YS, Batieha A, Ajlouni H, El-Khateeb M. An increase in prevalence of diabetes mellitus in Jordan over 10 years. J Diabetes Complications 2008;22:317-24.

7. Khader Y, Bateiha A, El-Khateeb M, Al-Shaikh A, Ajlouni K. High prevalence of the metabolic syndrome among Northern Jordanians. J Diabetes Complications 2007;21:214-9.

8. Khattab M, Khader YS, Al-Khawaldeh A, Ajlouni K. Factors associated with poor glycemic control among patients with type 2 diabetes. J Diabetes Complications 2010;24:84-9.

9. Lynch CJ, Adams SH. Branched-chain amino acids in metabolic signalling and insulin resistance. Nat Rev Endocrinol 2014;10:723-36.

10. Felig P, Marliss E, Ohman JL, Cahill CF Jr. Plasma amino acid levels in diabetic ketoacidosis. Diabetes 1970;19:727-8. 
11. Roberts LD, Koulman A, Griffin JL. Towards metabolic biomarkers of insulin resistance and type 2 diabetes: progress from the metabolome. Lancet Diabetes Endocrinol 2014;2:65-75.

12. Menni C, Fauman E, Erte I, Perry JR, Kastenmuller G, Shin SY, et al. Biomarkers for type 2 diabetes and impaired fasting glucose using a nontargeted metabolomics approach. Diabetes 2013;62:4270-6.

13. Lackey DE, Lynch CJ, Olson KC, Mostaedi R, Ali M, Smith WH, et al. Regulation of adipose branched-chain amino acid catabolism enzyme expression and cross-adipose amino acid flux in human obesity. Am J Physiol Endocrinol Metab 2013;304:E1175-87.

14. Pitkanen HT, Oja SS, Kemppainen K, Seppa JM, Mero AA. Serum amino acid concentrations in aging men and women. Amino Acids 2003;24:413-21.

15. Li YC, Li Y, Liu LY, Chen Y, Zi TQ, Du SS, et al. The ratio of dietary branched-chain amino acids is associated with a lower prevalence of obesity in young Northern Chinese adults: an Internet-Based CrossSectional Study. Nutrients 2015;7:9573-89.

16. Tabish SA. Is diabetes becoming the biggest epidemic of the twentyfirst century? Int J Health Sci (Qassim) 2007;1:V-VIII.

17. Mitu O, Mitu F, Leon MM, Roca M, Gherasim A, Graur M. Increased type 2 diabetes mellitus risk (assessed by Findrisc Score) is associated with subclinical atherosclerotic markers in asymptomatic adult population. Rom J Diabetes Nutr Metab Dis 2016;23:37-45.

18. Verges B. Pathophysiology of diabetic dyslipidaemia: where are we? Diabetologia 2015;58:886-99.

19. Lastra G, Syed S, Kurukulasuriya LR, Manrique C, Sowers JR. Type 2 diabetes mellitus and hypertension: an update. Endocrinol Metab Clin North Am 2014;43:103-22.

20. Newgard CB, An J, Bain JR, Muehlbauer MJ, Stevens RD, Lien LF, et al. A branched-chain amino acid-related metabolic signature that differentiates obese and lean humans and contributes to insulin resistance. Cell Metab 2009;9:311-26.

21. Hutson SM, Harper AE. Blood and tissue branched-chain amino and alpha-keto acid concentrations: effect of diet, starvation, and disease. Am J Clin Nutr 1981;34:173-83.

22. Tuso P. Prediabetes and lifestyle modification: time to prevent a preventable disease. Perm J 2014;18:88-93.

23. Layman DK. The role of leucine in weight loss diets and glucose homeostasis. J Nutr 2003;133:261S-267S. 